\title{
Tracer Norepinephrine Kinetics in Coronary Circulation of Patients with Heart Failure Secondary to Chronic Pressure and Volume Overload
}

\author{
C. P. Rose, J. H. Burgess, and D. Cousineau \\ McGill University Medical Clinic and Division of Cardiology, Department of Medicine, Montreal General Hospital, \\ 1650 Cedar Avenue, Montreal, Quebec H3G 1 A4
}

\begin{abstract}
Controversy exists over the nature of the abnormality in cardiac sympathetic nerves in heart failure. In the cardiomyopathy of the Syrian hamster, reduction in tissue stores and increased turnover of norepinephrine is clearly associated with excessive sympathetic stimulation but in animal models and humans with heart failure secondary to mechanical overload there is evidence for depression of neuronal uptake. Because norepinephrine is both released and taken up by sympathetic fibers it is impossible to assess norepinephrine kinetics in an intact heart without separating these two functions. A technique for doing so has recently been developed in normal dogs and we therefore acquired similar data in humans with heart failure secondary to chronic pressure and volume overload. The technique involves the combination of transient norepinephrine tracer coronary sinus outflow in relation to intravascular and interstitial references after simultaneous injection into the left coronary artery and the measurement of endogenous norepinephrine concentrations in artery and coronary sinus. We found a marked reduction in cardiac norepinephrine release and uptake in a group of patients with clinical left ventricular failure secondary to mechanical overload, relative to a group of patients with no failure. Norepinephrine balance and overflow across the heart were not significantly different. We conclude that there is hypofunction of the cardiac sympathetic nerves in heart failure secondary to mechanical overload and that traditional methods are inadequate in assessing cardiac norepinephrine kinetics when there are simultaneous changes in neuronal uptake and release.
\end{abstract}

\section{Introduction}

Chronic myocardial failure is associated with abnormalities in the sympathetic nervous system. Tissue levels of norepinephrine are reduced (1-4) in cardiac muscle and plasma levels are increased $(5,6)$. From studies on the cardiomyopathic Syrian hamster it has been surmised that cardiac tissue depletion in humans with chronic heart failure due to mechanical overload is due to increased sympathetic stimulation $(7,8)$. Indeed, Swedberg et al. $(9,10)$ observed increased positive aorto-coronary

Dr. Cousineau is a Scholar of the Medical Research Council of Canada, and Dr. Rose is a Chercheur Boursier of the Fonds de Recherche en Sante du Quebec. Address reprint requests to Dr. Rose, University Medical Clinic, Montreal General Hospital, 1650 Cedar Ave., Montreal, Quebec H3G 1A4, Canada.

Received for publication 27 February 1985 and in revised form 31 May 1985.

J. Clin. Invest.

(c) The American Society for Clinical Investigation, Inc.

$0021-9738 / 85 / 11 / 1740 / 08 \quad \$ 1.00$

Volume 76, November 1985, 1740-1747 sinus norepinephrine balance in human heart failure of various etiologies.

Since norepinephrine is both released and taken up by sympathetic nerve fibers it is impossible to assess the true neuronal release from simple measurement of arterial and coronary sinus norepinephrine concentrations. Thus, it is possible that both release and uptake could be reduced with little or no change in arterio-venous extraction. This hypothesis became more tenable when we showed in humans with heart failure secondary to chronic overload that sympathetic neuronal uptake of epinephrine, which is normally not released from peripheral sympathetic fibers, is reduced in the heart but not in the lower limb (11). Norepinephrine overflow was normal or increased. This observation confirmed several previous suggestions that in chronic heart failure secondary to mechanical overload there is a defect in the neuronal uptake and release that was specific to the heart and not part of a generalized exhaustion of the sympathetic system $(2,3,12)$.

Clearly what was needed was a nondestructive technique for measuring both neuronal uptake and release of norepinephrine in the intact, in situ human heart. Fortunately such an approach had been developed in the normal dog in our institution (13). Since the technique involves only analysis of transient tracer norepinephrine uptake in concert with endogenous arterial and coronary sinus norepinephrine concentrations, it is easily adapted to humans in the environment of the cardiac catheterization laboratory.

\section{Methods}

\section{Patient selection}

Patients referred to the cardiac catheterization laboratory of the Montreal General Hospital for routine diagnostic studies were selected and divided into two groups by the following criteria: group A had valve disease, predominantly mitral stenosis with no evidence of left ventricular hypertrophy on the electrocardiogram and no previous history of pulmonary edema; group B had valve disease with any combination of aortic stenosis, aortic insufficiency, or mitral insufficiency, electrocardiographic left ventricular hypertrophy, and a history of pulmonary edema confirmed by chest roentgenography. All patients were over the age of $40 \mathrm{yr}$ and therefore required routine coronary angiography to rule out significant coronary artery disease. None of the patients selected had significant coronary disease. Informed consent was obtained in a manner approved by the Clinical Trials Committee of the Montreal General Hospital. All medications were stopped $24 \mathrm{~h}$ before the catheterization. Anticoagulation with $5,000 \mathrm{U}$ of heparin was used in all patients.

\section{Catheterization}

After the routine diagnostic study a \#8F Judkins left coronary catheter was introduced into the aortic root and a \#6F Goodale-Lubin catheter was introduced into the coronary sinus via the right jugular vein. The position of the coronary sinus catheter was confirmed by contrast (MD76) injection and oxygen saturation of sampled blood. The Judkins catheter was then introduced into the ostium of the left coronary artery and its 
position was confirmed by an injection of contrast. The coronary sinus catheter was connected to a Sigmamotor pump that withdrew blood at a rate of $50-80 \mathrm{~cm}^{3}$ per minute from the coronary sinus. Immediately before each run simultaneous samples were drawn from the aorta and coronary sinus for assay of endogenous catecholamine concentrations.

\section{Multiple indicator dilution}

A run consisted of injecting $0.75 \mathrm{ml}$ of the injection mixture (see below) into the left coronary ostium while simultaneously collecting samples from the coronary sinus into heparinized tubes on a moving rack at a rate of $\sim 0.75 \mathrm{~s}$ per sample. Arterial pressure, heart rate, and electrocardiogram were monitored continuously. No change occurred in any of these parameters during the run in any patient.

\section{Injection mixture}

The injection mixture was composed of autologous blood adjusted to the hematocrit of each patient. It contained the following radioactive tracers: ${ }^{125}$ I-albumin (Charles E. Frosst, Pointe Claire, Quebec, Canada), a reference substance that does not leave the coronary circulation within a single passage; $\left[\mathrm{U}-{ }^{14} \mathrm{C}\right]$ sucrose (New England Nuclear, Boston, MA), a diffusible substance that leaves the circulation to enter the extracellular space during its passage through the coronary capillaries; and $\mathrm{N}-[1,7-$ ${ }^{3} \mathrm{H}$ ]norepinephrine (sp act, $30.0 \mathrm{Ci} / \mathrm{mol}$, New England Nuclear).

\section{Sample analysis}

A sample of $0.1 \mathrm{ml}$ from each heparinized tube collected during the run was diluted with $1.5 \mathrm{ml}$ of saline, pipetted into a counting tube, and assayed for radioactivity in a gamma ray spectrometer set for the photopeak characteristic of ${ }^{125} \mathrm{I}$. The proteins were then precipitated with $0.2 \mathrm{~mol}$ of trichloroacetic acid, and $0.2 \mathrm{ml}$ of the supernatant fluid was pipetted into a scintillation cocktail and assayed for ${ }^{14} \mathrm{C}$ and ${ }^{3} \mathrm{H}$ activity in a liquid-scintillation counter. Samples from the injection mixture, diluted with blood, and crossover standards were treated identically. To compare the relative magnitudes of the three tracers at the outflow, the activity in each sample was normalized by dividing the activity by the respective concentration in the injection mixture. The resulting value is the outflow fraction of the total injected per milliliter of venous blood.

For determination of catecholamine levels, $3 \mathrm{ml}$ of blood was transferred to ice-cold tubes that contained glutathione and EGTA, as described by Peuler and Johnson (14). Norepinephrine and epinephrine levels determined in duplicate in $50 \mu$ lof plasma by the radioenzymatic method outlined by these authors with only minor modifications (Cat-a-Kit, Upjohn Co., Kalamazoo, MI).

\section{Qualitative description of data}

Normalized coronary sinus outflow curves obtained in patients from the two groups are shown in Fig. 1. Initial insight into this data's meaning is aided by consideration of the events occurring in a single capillary as the bolus of tracers travels along it. Since labeled albumin does not leave the capillaries in a single passage to any significant extent, the time of its outflow is determined solely by the transit time of the capillary. In the whole organ there will of course be a distribution of pathways connecting inlet and outlet. Labeled sucrose occupies the same plasma space as albumin but also permeates the capillary barrier via aqueous channels and enters the interstitial space. It does not cross any cell membranes and is therefore a reference for the interstitial space. In the early samples the sucrose curve is reduced in relation to the albumin curve because of diffusion from the capillary, but later, as labeled sucrose returns from the interstitial space, its concentration is higher than that of albumin, which has already left the capillary. Labeled norepinephrine is handled in a similar fashion at the capillary surface but its removal and concentration by the sympathetic fibers in the interstitial space, which run parallel to the capillaries, reduces the amount returning from the interstitial space. Since Chidsey et al. (15) have shown that 3 min after injecting labeled norepinephrine into the coronary artery of the canine heart, the metabolites of norepinephrine constitute only a barely perceptible proportion of the total radioactivity measurable in the coronary sinus, we have as-

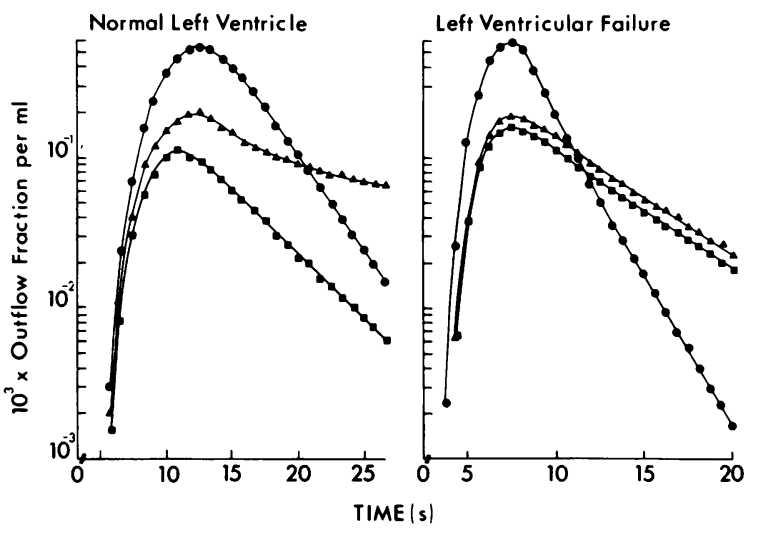

Figure 1. Representative normalized coronary sinus outflow curves from a patient from group A with mitral stenosis (left) and a patient from group B with aortic stenosis, aortic regurgitation, mitral regurgitation, left ventricular hypertrophy, and documented pulmonary edema (right). See text for qualitative interpretation of these patterns. •, ${ }^{125} \mathrm{I}$-albumin; $\Delta,\left[{ }^{14} \mathrm{C}\right]$ sucrose; $\boldsymbol{\varpi},\left[{ }^{3} \mathrm{H}\right]$ norepinephrine.

sumed that the tritiated material obtained at the outflow in our experiments over periods up to $40 \mathrm{~s}$ has remained unchanged.

In a representative patient without clinical heart failure or left ventricular hypertrophy (Fig. 1, left), there is a such a large extraction of labeled norepinephrine that its outflow concentration never rises above the labeled albumin curve. This pattern is identical to that found in the normal dog. In a patient with clinical heart failure secondary to chronic aortic and mitral valve disease, left ventricular hypertrophy and dilated heart (Fig. 1, right), there is a marked reduction in labeled norepinephrine sequestration and the pattern of outflow is close to that of sucrose. This pattern is reminiscent of that obtained after inhibition of neuronal uptake by desmethylimipramine in the dog (13). While inspection of the data suggests that cardiac neuronal uptake is decreased in patients with heart failure, it is only possible to quantitate the reduction and calculate the norepinephrine release by means of a mathematical model of the bloodtissue exchange process.

\section{Mathematical modeling}

Transient tracer outflow. The assumptions underlying and the description of the model of blood-tissue exchange of sequestered substances in the coronary circulation as well as the methods for its calculation have been described extensively in previous publications $(13,16-19)$, but the main points are outlined here so that the reported parameters can be related to their position in the equations of the model. First, a single capillary model is derived and then this is inserted into a whole-organ model with variable capillary transit times. Fig. 2 shows diagrammatically the single capillary model for irreversible sequestration of norepinephrine by sympathetic neurons in the interstitial space. The assumptions corresponding to this are that $(a)$ dispersionless plug flow occurs in the capillary; $(b)$ longitudinal diffusion can be neglected because the axial dimension is very large with respect to the radial dimension; (c) radial diffusional equilibration is so rapid that no concentration gradients exist in this direction within any of the spaces; $(d)$ substrate sequestration takes place with first-order kinetics.

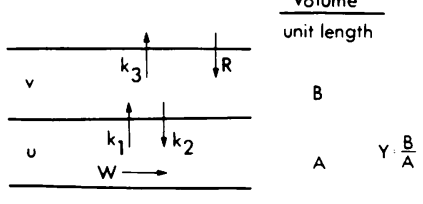

Figure 2. Diagramatic representation of the single-capillary mathematical model employed in the analysis of the coronary sinus outflow curves and the estimation of bulk uptake and release. Symbols are defined in the text. 
In the modeling and in the illustration the following symbols are used: $A$ and $B$ are the volumes per unit length for capillary and interstitial spaces, respectively; $\mathrm{W}$ is the velocity of blood flow; $u(x, t)$ and $v(x, t)$ are, respectively, tracer concentrations at some point, $x$, along the length of the unit and at time, $t ; k_{1}$ and $k_{2}$ are permeability-surface products per unit interstitial space for flux out of and into the capillary, respectively; $k_{3}$ is the first-order unidirectional rate constant per unit interstitial space for irreversible sequestration by the neuron; and $R$ is the rate of release of unlabeled norepinephrine from the neuronal pool.

Consideration of the events occuring at each element in space and time leads to two partial differential equations that must be solved simultaneously. The first for the conservation of matter is:

$\frac{\partial u}{\partial t}+\frac{\partial u}{\partial x}+\gamma \frac{\partial u}{\partial t}=0$

where $\gamma=\mathrm{B} / \mathrm{A}$

The second, the rate equation for accumulation of tracer in the interstitial space, is:

$\frac{\partial u}{\partial t}=k_{1} u-k_{2} u-k_{3} u$

Suppose that an amount of tracer $\left(q_{0}\right)$ is injected at the origin of capillary of length $L$ with flow $F_{c}$. Define the Laplace transform $(\xi)$ of the outflow concentration of label as:

$\mathrm{U}(\mathrm{L}, s)=\int_{0} \mathrm{U}(\mathrm{L}, t) \mathrm{e}^{-s t} d t=\mathfrak{£}[u(\mathrm{~L}, t)]$,

where $s$ (the domain of $\varepsilon$ ) is a complex plane corresponding to the transport function for the reference tracer.

Then, from the partial differential equations,

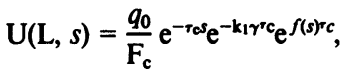

where $f(s)=\gamma k_{1} k_{2} /\left\{s+k_{2}+k_{3}\right\}$ and $\tau_{\mathrm{c}}=\mathrm{L} / \mathrm{W}$, which is the transit time for the flow of materials confined to the capillary. The inversion of this transform gives a time-domain solution of the form,

$u(\mathrm{~L}, t)=\frac{q_{0}}{\mathrm{~F}_{\mathrm{c}}} \mathrm{e}^{-k_{1} \gamma^{\prime} c} \delta\left(t-\tau_{c}\right)+$ returning component

where $\delta$ symbolizes the Dirac delta or impulse function. The first term is the "throughput" or "nonexchanging" component and represents an initial impulse arriving at the outflow concurrently with the vascular reference but reduced in magnitude by the factor $\exp \left(-k_{1} \gamma \tau_{c}\right)$. Note that $k_{1} \gamma \tau_{\mathrm{c}}$ can also be written as $\mathrm{PS} / \mathrm{F}_{\mathrm{c}}$, where $\mathrm{P}$ is the permeability of the capillary and $S$ is its surface area. The second term, the "returning component," describes the outflow of material that permeated the wall of the capillary and then returns to it after the throughput has exited. These two components correspond roughly to the peak and downslope of the whole-organ outflow. The expression for the returning component is particularly complicated and the interested reader is asked to consult the above references.

The construction of the whole-organ model requires three more assumptions: (e) the myocardial circulation is composed of groups of capillaries with common transit times; $(f)$ the ratio of interstitial space size to capillary volume is constant for all groups of capillaries; and $(g)$ capillaries and nonexchanging vessels are connected in such a way that more rapid capillary transit times are associated with more rapid large-vessel transit times. Flow coupling (flow of material from an arteriole to its group of capillaries) would be expected to result in this kind of effect.

With these assumptions the impulse response of the whole organ in the frequency domain is:

$\mathrm{C}(s)=\mathrm{e}\left\{\left[-k_{1} \gamma+f(s)\right] \tau_{c, \text { min }}\right\} \mathrm{W}_{0}\left[s+k_{1} \gamma b-b f(s)\right]$,

where $\tau_{c, \text { min }}$ is the minimum capillary transit time, $b$ is the linear factor relating the capillary transit time to the elapsed time after $\tau_{c, \min }, \mathrm{W}_{\mathrm{o}}(s)$ is the impulse response of the reference tracer, and the capillary transit time

$\tau_{\mathrm{c}}(t)=\tau_{\mathrm{c}, \min }+\mathrm{b}\left(t-\tau_{\mathrm{c}, \min }\right) \quad 0 \leq b \leq 1$.

Note that in general $\gamma$ is not an independent parameter but in the wholeorgan transform is always combined with either $\tau_{\mathrm{c}, \min }$ or $b$. Also in practice it is not possible to obtain absolute capillary transit times, and the parameters $\tau_{\mathrm{c}, \min }$ and $b$ are replaced by $a^{\prime}=k_{1} \gamma \tau_{\mathrm{c}, \min }$, and $b^{\prime}=k_{1} \gamma b$, respectively.

Because real data are truncated, usually at the onset of recirculation, the transform of these data can be obtained only through extrapolation. Empirically outflow curves are known to always decrease monoexponentially in their final phase. To fit the data in the transform or frequency domain one last assumption is needed: $(h)$ all dilution curves can be extrapolated monoexponentially.

In the case of completely recovered permeating tracer such as sucrose, the downslope can be extrapolated so that its recovery matches that for the vascular reference, albumin. For norepinephrine whose recovery is necessarily less than complete, it is necessary to define a terminal slope by running a regression line through the last few data points. The extrapolated curves can then be fitted sequentially in the frequency domain. This is done by iterative adjustment of the model parameters until the predicted outflow of the permeating tracer matches the data as closely as possible given the form of the vascular reference in the same experiment.

A set of outflow curves such as those displayed in Fig. 1 yields six unique, optimized parameters and one model-independent parameter, which characterize the state of capillary exchange in the 20-30 s in which the tracers are passing through the coronary microcirculation. For sucrose, the optimized parameters are: $k_{s}\left(=k_{1}=k_{2}\right)$, the capillary permeabilitysurface product per unit interstitial space; and $a^{\prime}$ and $b^{\prime}$, which describe the heterogeneity of capillary transit times. For norepinephrine the parameters are: $k_{\mathrm{c}}\left(=k_{1}=k_{2}\right)$, the capillary permeability-surface product per unit interstitial space for norepinephrine; $k_{\mathrm{n}}\left(=k_{3}\right)$, the rate constant for unidirectional sequestration by the sympathetic neurons; and $\gamma$, the ratio of the accessible interstitial space for norepinephrine relative to sucrose. The model-independent parameter is phi, the plasma flow per unit interstitial space. It is calculated as the inverse of the difference in mean transit times between albumin and sucrose.

Steady-state uptake and release. These parameters can be used in combination with the endogenous catecholamine concentration to derive estimates of steady-state rates of neuronal uptake and release. It can be shown (13) that the norepinephrine concentration in the coronary sinus,

$$
\begin{aligned}
u_{c s} & =u_{0} \sum_{j=1}^{n} w_{\mathrm{j}}\left\{\exp \left(-\frac{k_{\mathrm{c}} k_{\mathrm{n}} \gamma \tau_{\mathrm{j}}(t)}{\left(k_{\mathrm{c}}+k_{\mathrm{n}}\right)}\right)\right. \\
& \left.+\frac{\mathrm{R}}{k_{\mathrm{n}}}\left[1-\exp \left(-\frac{k_{\mathrm{c}} k_{\mathrm{n}} \gamma \tau_{\mathrm{j}}(t)}{\left(k_{\mathrm{c}}+k_{\mathrm{n}}\right)}\right)\right]\right\},
\end{aligned}
$$

where $u_{0}$ is the arterial norepinephrine concentration, $R$ is the rate of generation of unlabeled norepinephrine in the interstitial space, $w$ is the fraction of the reference represented at each outflow time, such that the sum of the total $\sum_{j=1}^{n} w_{j}=1.0$; and $\gamma \tau_{j}(t)$ has been defined at each point along the tracer norepinephrine curve by fitting of the modeling to the outflow tracer dilution curve. The only unknown parameter is $\mathbf{R}$. With knowledge of input and output coronary sinus concentrations, it is then possible to insert into this expression an initial estimate of $R$, the steadystate rate of norepinephrine generation or release into the interstitial space per unit of interstitial space, and, from the initial input arterial unlabeled norepinephrine concentration, to predict a mixed venous or coronary sinus bulk norepinephrine concentration; and then to optimize the $R$ value until the predicted mixed venous concentration and the experimentally determined values coincide. These values can then be used to calculate the average interstitial norepinephrine concentrations for the whole organ. The interstitial norepinephrine concentration, $v$, at 
any point, $x$, along the capillary is related to the plasma concentration by the relation,

$v(x)=\frac{k_{\mathrm{c}} u(x)}{\left(k_{\mathrm{c}}+k_{\mathrm{n}}\right)}+\frac{\mathrm{R}}{\left(k_{\mathrm{c}}+k_{\mathrm{n}}\right)}$.

Bulk steady-state norepinephrine uptake is then $=\mathrm{R}-\operatorname{phi}\left(C_{\text {ven }}\right.$ $-C_{\text {art }}$.

We have also taken the opportunity to compare our model-dependent estimates of norepinephrine kinetics with less rigorous methods. The plasma norepinephrine extraction is $\left(C_{\text {ven }}-C_{\text {arr }}\right) / C_{\text {arr }}$. The norepinephrine balance, phi $\left(C_{\text {ven }}-C_{\mathrm{arr}}\right) / C_{\mathrm{art}}$, requires only the measurement of coronary flow but completely ignores the existence of simultaneous uptake and release. The norepinephrine overflow $=\operatorname{phi}\left\{C_{\text {ven }}-C_{\text {art }}(1-E)\right\}$, where $E$ is the steady-state extraction of labeled norepinephrine, and has been recommended as a means of correcting for simultaneous norepinephrine uptake (20). The steady-state extraction can be estimated from our data by using the ratio of the difference between the areas subtended by the albumin and norepinephrine curves to the area under the albumin curve. However, this calculation still ignores the influence of the capillary barrier on the exchange process. Even if all norepinephrine were completely removed from the interstitial space by the neurons, $30-40 \%$ of the arterial input would appear in the coronary sinus (17). Thus net overflow is relatively insensitive to alterations in neuronal release and uptake.

Differences between means for each parameter in the two groups were determined by the unpaired $t$ test. The null hypothesis was rejected at the level of 0.05 .

\section{Results}

Table I shows clinical data, endogenous norepinephrine concentrations, coronary sinus oxygen saturation, phi (plasma flow per unit interstitial space), and norepinephrine balance and labeled norepinephrine extraction for each patient in the two groups. The only difference was a significant reduction in the labeled norepinephrine extraction in the heart failure group (33\% vs. $73 \%$ in group A). As in our previous study (11), the aortocoronary sinus epinephrine extraction was negligible in the failure group.

Table II and Fig. 3 show parameters derived from the model. The only variable differing significantly between the two groups was the rate constant for neuronal uptake, which was reduced by $90 \%$ in group B.

Table II and Fig. 4 compare steady-state estimates of norepinephrine kinetics with estimates of neuronal uptake and release calculated using the model combining transient kinetics and endogenous levels. Neither norepinephrine balance nor overflow give any hint of the rather gross abnormalities in both uptake and release revealed by the more complete analysis.

Fig. 5 compares norepinephrine release and uptake in all patients. The diagonal line is the line of identity. A remarkable equality exists in the two functions of the sympathetic nerves. On the average, there is slightly more release than uptake but this is not constant and is not related to the state of the left ventricle.

Fig. 6 correlates the calculated cardiac norepinephrine release with the norepinephrine concentration in the coronary sinus, a crude index of the degree of peripheral sympathetic stimulation. For a given level of circulating norepinephrine, the group with left ventricular failure had much less cardiac release. This implies that there is a localized defect in the cardiac portion of the peripheral sympathetic nervous system.

\section{Discussion}

Definition of heart failure. Patients were assigned to the groups on the basis of clinical findings alone. While there is no direct relationship between left ventricular contractility and clinical

Table I. Plasma Catecholamines and Steady State Parameters

\begin{tabular}{|c|c|c|c|c|c|c|c|c|}
\hline Unit no. & Diagnosis & $\begin{array}{l}\text { NE arterial } \\
\text { concentration }\end{array}$ & $\begin{array}{l}\text { NE venous } \\
\text { concentration }\end{array}$ & $\begin{array}{l}\text { Plasma } \\
\text { NE } \\
\text { extract }\end{array}$ & $\begin{array}{l}\text { Tracer } \\
\text { NE } \\
\text { extract }\end{array}$ & Phi & NE balance & $\begin{array}{l}\text { NE } \\
\text { overflow }\end{array}$ \\
\hline & & nmol & nmol & & & $s^{-1}$ & $\mathrm{pmol} / \mathrm{ml} / \mathrm{s}$ & $\mathrm{pmol} / \mathrm{ml} / \mathrm{s}$ \\
\hline \multicolumn{9}{|l|}{ Group A } \\
\hline 774648 & MS & 0.710 & 0.860 & -0.211 & 0.686 & 0.057 & 0.0086 & 0.0366 \\
\hline 162617 & MS, MR & 2.890 & 4.880 & -0.689 & 0.712 & 0.064 & 0.1282 & 0.2607 \\
\hline 755427 & MS & 2.910 & 2.640 & 0.093 & 0.755 & 0.040 & -0.0109 & 0.0777 \\
\hline 789584 & MS & 2.960 & 2.490 & 0.159 & 0.792 & 0.053 & -0.0248 & 0.0988 \\
\hline 646114 & MR & 1.800 & 1.290 & 0.283 & 0.656 & 0.039 & -0.0199 & 0.0262 \\
\hline 665603 & MS, AR & 1.680 & 1.540 & 0.083 & 0.781 & 0.044 & -0.0061 & 0.0515 \\
\hline Average & & 2.158 & 2.283 & -0.047 & 0.730 & 0.050 & 0.013 & 0.092 \\
\hline $\mathrm{SD}$ & & 0.836 & 1.321 & 0.323 & 0.50 & 0.009 & 0.053 & 0.079 \\
\hline \multicolumn{9}{|l|}{ Group B } \\
\hline 198914 & AS, AR, MR & 3.840 & 2.110 & 0.451 & 0.414 & 0.025 & -0.0434 & -0.0035 \\
\hline 351542 & MR, MS & 2.380 & 6.320 & -1.655 & 0.247 & 0.036 & 0.1430 & 0.1644 \\
\hline 774647 & MR & 0.990 & 1.580 & -0.596 & 0.327 & 0.060 & 0.0353 & 0.0547 \\
\hline 104142 & AS, AR, MR & 6.140 & 7.950 & -0.295 & 0.160 & 0.048 & 0.0860 & 0.1326 \\
\hline 481175 & $\mathrm{AR}, \mathrm{MR}$ & 1.250 & 1.270 & -0.016 & 0.489 & 0.030 & 0.0006 & 0.0189 \\
\hline 779210 & MR, AS, AR & 4.030 & 6.360 & -0.578 & 0.318 & 0.071 & 0.1659 & 0.2571 \\
\hline Average & & 3.105 & 4.265 & -0.448 & $0.326^{*}$ & 0.045 & 0.065 & 0.104 \\
\hline SD & & 1.782 & 2.678 & 0.647 & 0.107 & 0.016 & 0.075 & 0.090 \\
\hline
\end{tabular}

* $P<0.05$ compared with mean of group A. AR, aortic regurgitation; AS, aortic stenosis; MR, mitral regurgitation; MS, mitral stenosis; NE, norepinephrine. 


\begin{tabular}{|c|c|c|c|c|c|c|c|c|c|}
\hline Unit no. & $k_{4}$ & $\mathbf{b}^{\prime}$ & $a^{\prime}$ & $\gamma$ & $k_{e}$ & $k_{\mathbf{z}}$ & $\begin{array}{l}\mathrm{NE} \\
\text { release }\end{array}$ & $\begin{array}{l}\text { NE } \\
\text { uptake }\end{array}$ & $\begin{array}{l}\text { NE interstitial } \\
\text { concentration }\end{array}$ \\
\hline & $s^{-1}$ & $s^{-1}$ & $s^{-1}$ & & $s^{-1}$ & $s^{-1}$ & $\mathrm{pmol} / \mathrm{ml} / \mathrm{s}$ & $\mathrm{pmol} / \mathrm{ml} / \mathrm{s}$ & nmol \\
\hline \multicolumn{10}{|l|}{ Group A } \\
\hline 774648 & 0.079 & 0.046 & 1.079 & 1.278 & 0.117 & 0.221 & 0.159 & 0.1504 & 0.870 \\
\hline 162617 & 0.103 & 0.533 & 0.862 & 2.161 & 0.147 & 0.323 & 0.836 & 0.7078 & 5.070 \\
\hline 755427 & 0.058 & 0.092 & 0.547 & 1.860 & 0.090 & 0.397 & 0.513 & 0.5239 & 2.540 \\
\hline 789584 & 0.079 & 0.024 & 0.966 & 1.864 & 0.154 & 0.604 & 0.770 & 0.7948 & 2.450 \\
\hline 646114 & 0.054 & 0.000 & 1.302 & 1.109 & 0.077 & 0.147 & 0.143 & 0.1629 & 1.230 \\
\hline 665603 & 0.095 & 0.110 & 0.990 & 1.235 & 0.131 & 0.287 & 0.351 & 0.3571 & 1.540 \\
\hline Average & 0.089 & 0.134 & 0.958 & 1.584 & 0.119 & 0.330 & 0.462 & 0.449 & 2.268 \\
\hline SD & 0.018 & 0.182 & 0.228 & 0.393 & 0.028 & 0.145 & 0.272 & 0.249 & 1.383 \\
\hline \multicolumn{10}{|l|}{ Group B } \\
\hline 198914 & 0.035 & 0.000 & 1.624 & 1.729 & 0.046 & 0.030 & 0.014 & 0.0575 & 2.080 \\
\hline 351542 & 0.052 & 0.000 & 1.257 & 1.566 & 0.071 & 0.019 & 0.188 & 0.0450 & 6.560 \\
\hline 774647 & 0.062 & 0.000 & 1.030 & 1.550 & 0.091 & 0.063 & 0.080 & 0.0445 & 1.600 \\
\hline 104142 & 0.054 & 0.000 & 1.137 & 1.345 & 0.063 & 0.015 & 0.158 & 0.0717 & 9.090 \\
\hline 481175 & 0.060 & 0.080 & 0.886 & 1.242 & 0.095 & 0.056 & 0.058 & 0.0569 & 1.270 \\
\hline 779210 & 0.073 & 0.000 & 0.923 & 1.440 & 0.128 & 0.062 & 0.417 & 0.2513 & 6.370 \\
\hline Average & 0.056 & 0.013 & 1.143 & 1.479 & 0.082 & $0.041^{*}$ & $0.052 \dagger$ & $0.088 \dagger$ & 4.495 \\
\hline SD & 0.012 & 0.030 & 0.249 & 0.159 & 0.026 & 0.020 & 0.132 & 0.074 & 2.986 \\
\hline
\end{tabular}

* $P<0.05$ compared with mean of group A.

findings, there is also no generally accepted unequivocal index of contractility, particularly in the presence of regurgitant valve lesions. We therefore assumed that those patients with electrocardiographic left ventricular hypertrophy and at least one episode of radiologic pulmonary edema have reduced left ventricular contractility secondary to chronic mechanical overload. Even with this imprecise definition every one of the patients in group B had a rate constant for neuronal uptake of norepinephrine far below the range in group $A$. Interestingly, the means of the model parameters for group $\mathrm{A}$ are with minor variations identical to those for the normal $\operatorname{dog}(13)$.

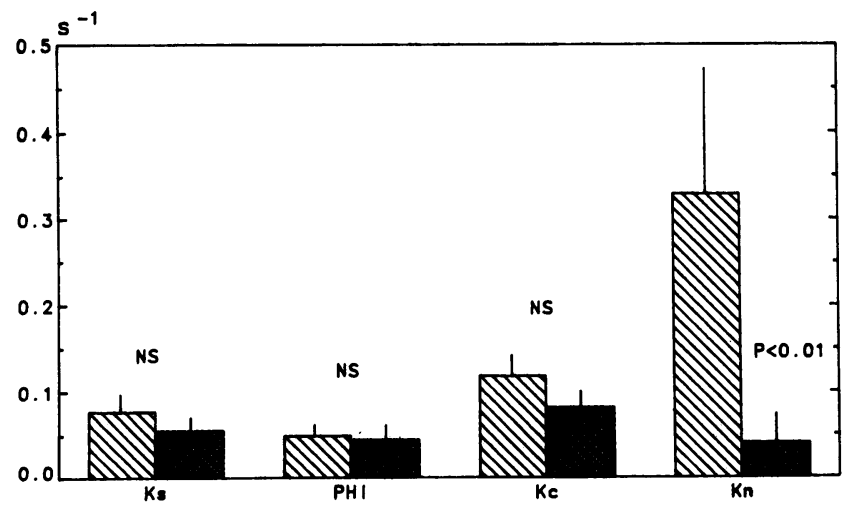

Figure 3. Rate constants derived from the model for tracer sucrose and norepinephrine. $k_{\mathrm{s}}$ and $k_{\mathrm{c}}$ are the rate constants for capillary exchange for sucrose and norepinephrine, respectively; phi is the plasma flow per unit interstitial space, and $K_{\mathrm{n}}$ is the rate constant for unidirectional uptake by the sympathetic neurons. $₫$, no failure; $\square$, failure.
Effect of hypertrophy and failure on interstitial space size. Because the total amount of tracer entering the coronary ostium is not known with certainty, the multiple indicator dilution technique as employed in this study allows estimation of the transport parameter relative to the interstitial space. If there were major changes in interstitial space size in hypertrophy, this would be reflected in an artifactual reduction in the rate constant for norepinephrine sequestration, $k_{\mathrm{n}}$. Fuster et al. (21) found only a nonsignificant increase in interstitial space size $(35.7 \%)$ in the autopsied left ventricle of patients with mitral regurgitation and moderate heart failure. There was a $50 \%$ increase in interstitial

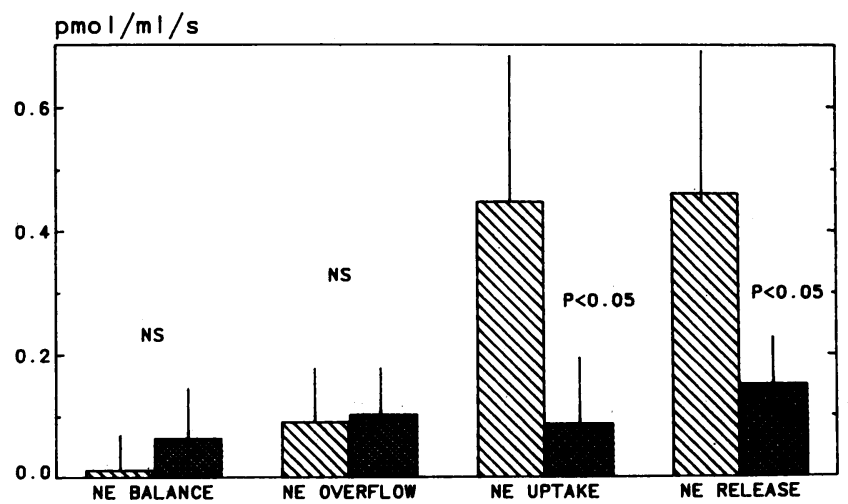

Figure 4. Comparison of methods for calculating norepinephrine kinetics. Steady-state calculations (balance and overflow) do not reveal the great reduction in uptake and release apparent with the transient technique. See text for methods of calculation. $₫$, no failure; $\mathbf{n}$, failure. 


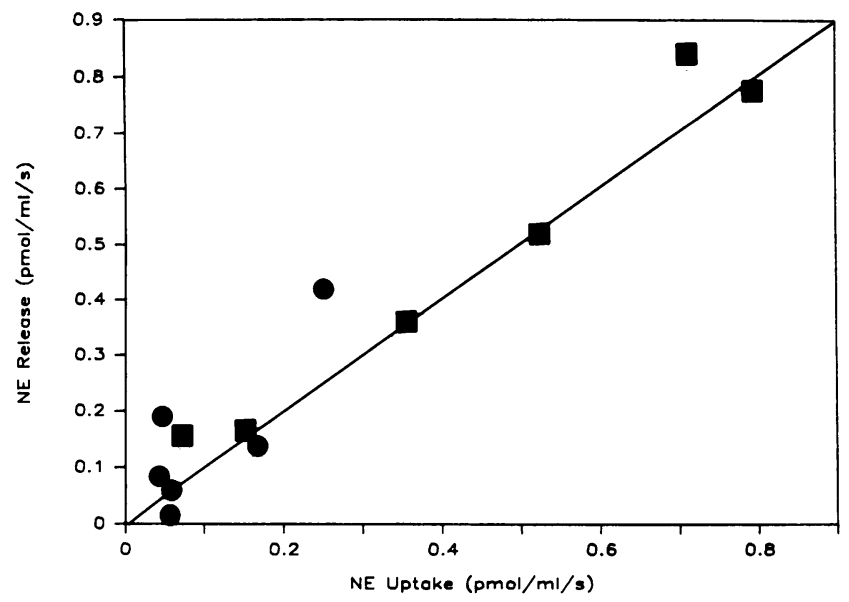

Figure 5. Correlation of norepinephrine release and norepinephrine uptake in all patients. Squares are group A and circles are group B. The diagonal line is the line of identity.

space size in patients with end-stage heart failure secondary to mechanical overload. The patients in group B of the present study had mild to moderate failure. To explain a $90 \%$ reduction in the rate constant for norepinephrine sequestration, the interstitial space size would have had to increase by a factor of 10 . Thus, we believe that most of the change observed is a real reduction in nerve uptake.

Comparison with other measurements of norepinephrine $\mathrm{ki}$ netics. By using the multiple indicator dilution technique we have been able to show depression of both uptake and release functions of sympathetic nerves in the intact hearts of humans with heart failure secondary to mechanical overload. It is not surprising that measurement of norepinephrine balance does not reveal this change because it does not take into consideration the simultaneous uptake and release. Unlike some previous studies $(9,10)$, we did not find a significant positive balance in the failure group. Net release is probably only seen during sympathetic stimulation and this would be expected to be highly variable.

The inability of norepinephrine overflow (spillover), which uses steady-state tracer extraction to correct for uptakę, to detect the change in neuronal release requires an explanation. While

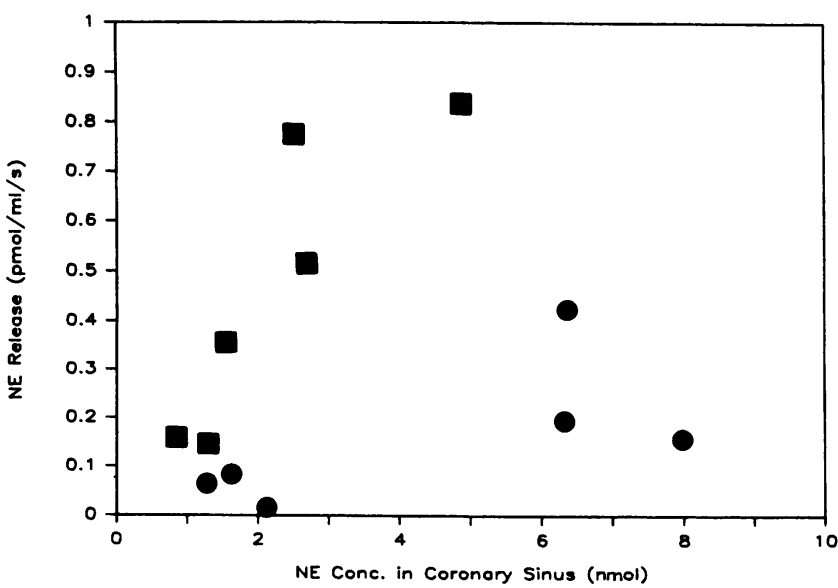

Figure 6. Norepinephrine release vs. norepinephrine concentration in the coronary sinus. Symbols are the same as in Fig. 5. there was a significant reduction in the calculated steady-state extraction, it was small relative to the change in $k_{\mathrm{n}}$, the rate constant for neuronal sequestration (twofold vs. ninefold); the transient analysis takes into account the fraction of tracer, which never leaves the capillary (30-40\%). Thus, in the presence of a relatively large barrier to blood-tissue exchange, the overflow calculation is just not sensitive enough. Presumably, if the uptake were zero or extremely low, say in a totally denervated heart, calculated overflow would then possibly decrease.

Comparison of tracer norepinephrine and endogenous epinephrine. This thought raises the question of why there is a zero extraction of epinephrine in the failure group whereas the tracer norepinephrine extraction is $33 \%$ on the average. The likely explanation is that the uptake system has less affinity for epinephrine than for norepinephrine. Indeed, the normal endogenous epinephrine extraction is only $50 \%(11,22)$ compared with the normal tracer norepinephrine extraction of $73 \%$. Final resolution of this question will require tracer epinephrine data.

Relation of uptake to release. Fig. 5 shows that norepinephrine release and uptake tend to be very closely matched in this relatively unstimulated selection of patients. It is natural to wonder if either is the primary event or if these functions are both affected independently by some other factor. Prolonged suppression of uptake could theoretically result in tissue depletion and reduced release but, vice versa, vesicular depletion by reserpine is known to cause reduced uptake (23). Alternately, both functions could be suppressed by a general abnormality in neuronal metabolism or destruction of nerves associated with the failure state.

Studies in animal models of overload heart failure have shown that despite reduced tissue concentration, the turnover of norepinephrine is normal (24). In this light our observation that release is decreased in proportion to uptake implies that on the average what we are seeing is a total loss of these functions in $80-90 \%$ of the sympathetic neurons adjacent to the capillaries in the left ventricle and relatively normal function in the remainder. This concept is supported by the observations of Borchard (25), who measured the density of autonomic nerves in atria from patients with heart failure and found a reduction or "thinning" not explained by myocyte hypertrophy. However, it is impossible to rule out reduction in nerve function without anatomical disappearance using only the multiple indicator dilution technique, and it is possible that both mechanisms could be operative simultaneously or at different times in the development of heart failure.

Location of impaired neurons and tissue depletion. Because we have used left coronary injection and coronary sinus sampling we do not see nerves in the right ventricle or the atria. However, biopsies of right atria in humans during valve replacement also show decreased tissue levels $(12,26,27)$. Animal models of right or left ventricular overload also show tissue depletion in the stressed ventricle first followed by depletion in the unstressed ventricle and the atria $(28,29)$. However, the preganglionic elements are probably intact because tyrosine hydroxylase activity is normal in the stellate ganglion (30). This constellation of observations can be interpreted by hypothesizing that sympathetic neurons first become impaired in the stressed ventricle but later the postsynaptic neurons degenerate retrogradely. Destruction of sympathetic nerve terminals by repeated injections of 6-hydroxydopamine in adult rabbits is associated with an $80 \%$ reduction in the ganglionic cell bodies in the superior cervical ganglion (31). Such a reduction in ganglionic cell bodies may 
also occur in patients with heart failure secondary to mechanical overload but has not been reported as yet.

Cardiac norepinephrine release and circulating catecholamines. Fig. 6 shows that as well as a significant difference in the mean norepinephrine release in the two groups, there is a more striking difference when release is related to coronary sinus norepinephrine concentration, a crude index of degree of sympathetic stimulation. Evidence for depression of the uptake function of the nerves in animal models $(2,3)$ and in human autopsy material (12) has been obtained previously. It may be deduced that if there were no substantial net positive balance of endogenous norepinephrine across the heart, neuronal release would have to decrease in concert with the decreased uptake, but no direct evidence for this had been obtained previously. Indeed, we have shown that it is only with detailed analysis of the transient kinetics of blood-tissue exchange that one can show such a phenomenon. Even the use of steady-state labeled norepinephrine extraction is not sensitive enough to detect the marked inhibition of release.

Congestive heart failure is known to be associated with increased circulating catecholamines $(5,25)$. This study raises the obvious question of the origin of these increased levels if the heart is releasing much less than usual for a given concentration in the coronary sinus. In the absence of any evidence that stimulation of cardiac nerves can be specifically inhibited during massive stimulation of the peripheral sympathetic nervous system such as occurs with heart failure, we must conclude either that in heart failure all of the circulating catecholamines are coming from the adrenal medulla, or that there is a localized defect in the cardiac nerves. Because the adrenal medulla secretes mostly epinephrine and because circulating epinephrine-to-norepinephrine ratio is not increased in heart failure (25), the increased levels must be due to stimulation of extracardiac peripheral nerves. The concept of localized cardiac sympathetic dysfunction in heart failure is supported by a previous study from this institution that showed normal epinephrine extraction across the lower limb in spite of absent epinephrine extraction across the heart in patients with heart failure (11) and by studies in animal models of heart failure in which tissue levels and uptake were decreased in the heart but not in the kidney $(2,3)$.

Interstitial norepinephrine concentration. Bristow's group (32) has observed a reduction in beta-adrenergic receptor density in failing human hearts removed during heart transplantation. Since they also observed a localized reduction of receptors in the right ventricle of patients with right heart failure secondary to pulmonary hypertension (33), they proposed that a localized defect in uptake would cause increased interstitial norepinephrine concentration and consequent down regulation of beta-adrenergic receptors. Of course the alternative explanation is that failing muscle cells do not make the normal complement of receptors. In the present study the patients with heart failure secondary to valve disease did not have significantly elevated calculated average interstitial norepinephrine concentration but neither did they have elevated circulating levels. Note that interstitial concentration is a complicated function of cardiac neuronal release and uptake and circulating levels. This problem can only be answered by combining the analysis of norepinephrine kinetics as reported here with measurement of beta receptor density in either operative or transvenous biopsies.

Conclusions and significance. We have shown that patients with relatively mild left ventricular failure (no increase in circulating norepinephrine concentration) have defective cardiac sympathetic innervation. This defect must be confined to the heart because circulating levels are normal. The underlying cause of this association between failing cardiac myocytes and defective nerve function must remain speculative at present. There are two possibilities: failing myocytes could release a toxic substance that injures the adjacent axons; or both myocytes and axons could be exposed to the same detrimental external factor (hypoxia?). This finding could potentially form the basis of a test for early heart failure if it could be shown that the nerves are affected early in the disease and a suitable imaging agent could be found that is taken up by the same axonal transport system as norepinephrine.

\section{Acknowledgments}

The authors wish to express their appreciation to Louise Gagnon, Eva Ibrahim, Kay Lumsden, Ruth Oake, Stan Gunaratnam, and Steven Sze for their expert technical assistance; to Mario Talajic, Michele Turek, and Rose Tannous for their clinical assistance, and to Nancy Firth for typing the manuscript.

This work was supported by the Medical Research Council of Canada and the Quebec Heart Foundation.

\section{References}

1. Chidsey, C. A., E. H. Sonneblick, A. G. J. Morrow, and E. Braunwald. 1966. Norepinephrine stores and contractile force of papillary muscle from the failing human heart. Circulation. 33:43-51.

2. Fisher, J. E., W. D. Horst, and I. J. Kopin. 1965. Norepinephrine metabolism in hypertrophied rat hearts. Nature (Lond.). 207:951-953.

3. Spann, J. F., C. A. Chidsey, P. E. Pool, and E. Braunwald. 1965. Mechanism of norepinephrine depletion in experimental heart failure produced by aortic constriction in the guinea pig. Circ. Res. 17:312321.

4. Spann, J. F., C. A. Chidsey, and E. Braunwald. 1964. Reduction of cardiac stores of norepinephrine in experimental heart failure. Science (Wash. DC). 145:1439-1441.

5. Cohn, J. N., T. B. Levine, M. T. Olivari, G. S. Francis, and M. R. Bristow. 1984. Plasma norepinephrine as a guide to prognosis in patients with chronic congestive heart failure. N. Engl. J. Med. 311:819823.

6. Spann, J. F. 1984. Functional changes in pathologic hypertrophy. In Growth of the Heart in Health and Disease. R. Zak, editor. Raven Press, New York. 421-466.

7: Sole, M. J., C. M. Lo, C. W. Laird, E. H. Sonnenblick, and R. J. Wurtman. 1975. Norepinephrine turnover in the heart and spleen of the cardiomyopathic Syrian hamster. Circ. Res. 37:855-862.

8. Sole, M. J., A. B. Kamble, and M. N. Hussain. 1977. Possible change in the rate-limiting step for cardiac norepinephrine synthesis in the cardiomyopathic Syrian hamster. Circ. Res. 41:814-817.

9. Swedberg, K., C. Viquerat, J. L. Rouleau, M. Roizen, B. Atherton, W. W. Parmley, and K. Chatterjee. 1984. Comparison of myocardial catecholamine balance in chronic congestive heart failure and in angina pectoris without failure. Am. J. Cardiol. 54:783-786.

10. Swedberg, K., A. Hjolwarson, and S. Holmberg. 1979. Effects of work and acute beta-receptor blockade on myocardial norepinephrine release in congestive cardiomyopathy. Clin. Cardiol. 2:424-430.

11. Rose, C. P., J. H. Burgess, and D. Cousineau. 1983. Reduced aortocoronary sinus extraction of epinephrine in patients with left ventricular failure secondary to long-term pressure or volume overload. Circulation. 68:241-244.

12. Petch, M. C., and W. G. Nayler. 1979. Uptake of catecholamines by human cardiac muscle in vitro. Br. Heart J. 41:336-339.

13. Cousineau, D., C. A. Goresky, G. G. Bach, and C. P. Rose. 1984. 
Effect of beta-adrenergic blockade on in vivo norepinephrine release in canine heart. Am. J. Physiol. 246:H283-H292.

14. Peuler, J. D., and G. A. Johnson. 1977. Simultaneous single isotope radioenzymatic assay of plasma norepinephrine, epinephrine, and dopamine. Life Sci. 21:625-636.

15. Chidsey, C. A., D. C. Harrison, and E. Braunwald. 1962. Release of norepinephrine from the heart by vasoactive amines. Proc. Soc. Exp. Biol. Med. 109:488-490.

16. Rose, C. P., C. A. Goresky, P. Belanger, and M. J. Chen. 1980. Effect of vasodilation and flow rate on capillary permeability surface product and interstitial space size in the coronary circulation. Circ. Res. 47:312-328

17. Cousineau, D., C. P. Rose, and C. A. Goresky. 1980. Labeled catecholamine uptake in the dog heart: interactions between capillary wall and sympathetic nerve uptake. Circ. Res. 47:329-338.

18. Cousineau, D., C. P. Rose, and C. A. Goresky. 1981. In vivo characterization of the adrenergic receptors in the working canine heart. Circ. Res. 49:501-510.

19. Rose, C. P., and C. A. Goresky. 1984. Interactions between capillary exchange, cellular entry, and metabolic sequestration processes in the heart. In Handbook of Physiology. Section 2: The Cardiovascular System, Vol. IV. E. M. Renkin and C. C. Michel, editors. American Physiological Society, Bethesda, MD. 781-798.

20. Esler, M., G. Jennings, P. Korner, P. Blombery, N. Scharias, and P. Leonard. 1984. Measurement of total and organ-specific norepinephrine kinetics in humans. Am. J. Physiol. 247:E21-E28.

21. Fuster, V., M. A. Danielson, R. A. Robb, J. C. Broadbent, A. L. Brown, and L. R. Elveback. 1977. Quantitation of left ventricular myocardial fiber hypertrophy and interstitial tissue in human hearts with chronically increased volume and pressure overload. Circulation. 55: 504-508.

22. Halter, J. B., K. O. Kelley, and K. L. Gould. 1982. Cardiac uptake and secretion of catecholamines during adrenergic stimulation in vivo. Am. J. Physiol. 243:E52-E58.

23. Graefe, K. H., H. Bonisch, and U. Trendelenberg. 1971. Timedependent changes in neuronal net uptake or noradrenaline after pre- treatment with pargyline and/or reserpine. Naunyn-Schmiedeberg's Arch. Pharmacol. 271:1-28.

24. Schmid, P. G., D. D. Lund, J. A. Davis, C. A. Whiteis, R. K. Bhatnagar, and R. Roskoski, Jr. 1982. Selective sympathetic neural changes in hypertrophied right ventricle. Am. J. Physiol. 243:H175$\mathrm{H} 180$.

25. Borchard, F. 1978. The adrenergic nerves of the normal and the hypertrophied heart. Normal and Pathological Anatomy. W. Bargmann and W. Doerr, editors. Georg Thieme, Publishers, Stuttgart, Federal Republic of Germany. 33:1-68.

26. Mairrer, W., R. Tschada, J. Mawthey, A. Ablasser, and W. Kubler. 1981. Catcholamines in patients with heart failure in Catecholamines and the Heart. W. Delius, E. Gerlach, H. Grobecker, W. Kubeer, editors. Springer Verlag, New York. 236-245.

27. Chidsey, C. A., E. Braunwald, A. G. Morrow, and D. T. Mason. 1963. Myocardial norepinephrine concentration in man: effects of reserpine and congestive heart failure. N. Eng. J. Med. 269:653-658.

28. Vogel, J. H. K., D. Jacobowitz, and C. A. Chidsey. 1969. Distribution of norepinephrine in the failing bovine heart. Circ. Res. 24: $71-84$

29. Krakoff, L. R., R. A. Buccino, J. F. Spann, and J. de Champlain. 1968. Cardiac catechol O-methyltransferase and monoamine oxidase activity in congestive heart failure. Am. J. Physiol. 215:549-552.

30. Schmid, P. G., D. D. Lund, and R. Roskoski, Jr. 1981. Efferent autonomic dysfunction in heart failure. In Disturbances in Neurogenic Control of the Circulation. F. M. Abboud, H. A. Fozzard, J. P. Gilmore, and D. J. Reis, editors. American Physiological Society, Bethesda, MD.

31. Fronek, K. 1980. Evaluation of long-term chemical sympathectomy in adult rabbits. Am. J. Physiol. 238:H527-H532.

32. Bristow, M. R., R. Ginsberg, W. Minobe, R. S. Cubicciotti, S. Sageman, K. Lurie, M. E. Billingham, D. C. Harrison, and E. B. Stinson. 1982. Decreased catecholamine sensitivity and beta-adrenergic receptor density in failing human hearts. N. Engl. J. Med. 307:205-211.

33. Bristow, M. R., R. Ginsberg, W. A. Minobe, D. C. Harrison, B. A. Reitz, and E. B. Stinson. 1982. Beta-adrenergic receptor measurements in normal and failing human right and left ventricle. Circulation. 66(Suppl. II):207. 